\title{
Unilateral Bulging on Pharyngeal Contraction
}

National Cancer Institute

\section{Source}

National Cancer Institute. Unilateral Bulging on Pharyngeal Contraction. NCI Thesaurus.

Code C127317.

A finding of unilateral bulging on pharyngeal contraction during swallowing. 